\title{
An artificial fish swarm algorithm based on membrane computing and its application in permutation flow shop scheduling problem
}

\author{
Ping Liu ${ }^{1, \mathrm{a}}$ Yueguang $\mathrm{Li}^{2, \mathrm{~b}}$ \\ ${ }^{1}$ Gansu Normal University for Nationalities, Hezuo, gansu, 747000, China \\ ${ }^{2}$ Gansu Normal University for Nationalities, Hezuo, gansu, 747000, China \\ aYueguangli7@163.com, ${ }^{\text {b } Y u e g u a n g l i 7 @ s o h u . c o m ~}$
}

Keywords: Artificial fish swarm algorithm; Membrane computing; Optimization; Simulation

\begin{abstract}
In order to enhance the performance of artificial fish swarm algorithm in solving optimization problem, this paper added the membrane computing to artificial fish swarm algorithm, an improved artificial fish swarm algorithm of solving permutation flow shop scheduling problem was proposed. Simulation results show that the improved algorithm has better feasibility and validity for solving permutation flow shop scheduling problem.
\end{abstract}

\section{Introduction}

The artificial fish swarm algorithm is proposed by Li Xiaolei ${ }^{[1]}$ in 2002, it is a concrete application of the thought of swarm intelligence ${ }^{[2]}$. Artificial fish swarm algorithm is a kind of bionic optimization algorithm through simulation of fish behavior in nature, four operators were simulated four kinds of behavior of artificial fish so as to achieve the effect of local optimization of the algorithm, and according to their status updates and the surrounding environment, make their own in the population to find out the global optimal value. The algorithm has a strong ability to adapt to the quasi solution problem, no need to provide too much information of the objective function, global optimization ability good. Many scholars on this novel bionic optimization algorithm proposed many improved methods, for example, the simplified mobile way of fish swarm algorithm ${ }^{[3]}$, using adaptive step size ${ }^{[4]}$, reduce the search domain ${ }^{[5]}$, which makes the algorithm have been improved in some aspects, but there are still operational speed is slow, easy to fall into local optimum, low convergence precision.

Membrane computing ${ }^{[6]}$ is proposed by Ghcorghe Paun in 1998, membrane computing is a computing model from the life of the individual cell level, its purpose is to simulate the mechanism of the cells of life individual treated compound to achieve the optimization calculation.

According to the operation speed is slow, low convergence precision, easy to fall into the local extremum of artificial fish swarm algorithm, simplify the foraging behavior, according to the difference factor adaptive adjustment of step, line of sight, congestion factor, the number of attempts and other parameters, and the use of membrane computing communication rules to keep population diversity, the paper proposed an improved artificial fish swarm algorithm based on membrane computing.

The permutation flow shop scheduling problem is a simplified model of many actual production scheduling problems. Although the process constraint of the model is relatively simple, but it has proven more than 3 machine of permutation flow shop scheduling problem is NP problem.

\section{Artificial fish swarm algorithm and the membrane computing}

\subsection{Artificial fish swarm algorithm}

Four operators of foraging, cluster, collision, moving randomly of artificial fish swarm algorithm are used for the optimization calculation. Among them, $X=(x 1, x 2, \ldots x n)$ expresses the individual state in the artificial fish populations, $\mathrm{Xi}(\mathrm{i}=1, \ldots, \mathrm{n})$ expresses the variables to be optimized; $\mathrm{Y}=\mathrm{f}(\mathrm{X})$ expresses the food concentration value of artificial fish individual, where $\mathrm{Y}$ is the value of the objective function; $\mathrm{Di}, \mathrm{j}=\|\mathrm{Xi}-\mathrm{Xj}\|$ expresses the distance between two 
fish; $\delta$ expresses delta crowded degree factor; Visual expresses the maximum distance of artificial fish can be perceived; Step expresses mobile step of the artificial fish; the default behavior of foraging behavior is random behavior, the default behaviors of swarm behavior and following behavior are foraging behavior.

\subsection{The algorithm description}

Procedure Artificial Fish Swarm Algorithm

AF_init();

While the value is not satisfied

\{AF_prey()

\{If the value is better

AF_move();

Else random_ move();

\}

AF_swarm()

\{If the centre value is better

AF_ move();

Else $\mathrm{AF}_{-}$prey();

\}

AF_follow()

\{If another value is better

AF_move();

Else AF_prey();

\}

\}

Get result;

End while

End Artificial Fish Swarm Algorithm

\subsection{Membrane computing}

Generally, a P system of degree $\mathrm{n}$ can be expressed as:

$\prod=\left(V, T, C, \mu w_{1}, \ldots, w_{m},\left(R_{1}, \rho_{1}\right), \ldots,\left(R_{m}, \rho_{m}\right)\right)$

Among them: $\mathrm{V}$ is the alphabet, where elements are called objects; $T \subseteq V$ is the output alphabet; $C \subseteq V-T$ is catalyst, its role is to assist some rules implementation in the process of the optimization, but the catalyst itself remains unchanged; $\mu$ expresses membrane structure, which contains the mmembranes, various membranes and regions with label set $H, H=\{1,2, \ldots, m\}$, where $m$ is called degree of $\prod$; the two tuple $(\mu, v)$ represents the evolutionary rule.

\section{Artificial fish swarm algorithm based on membrane computing}

\subsection{Parameter analysis}

When the step size of step is larger, the convergence speed of fish is faster, but the convergence precision of fish is low; on the contrary, when the step size of step is small, the accuracy of convergence of fish swarm algorithm can be improved, but the convergence speed will slow down; the visual is bigger, the artificial fish can have large search range, it advantageous to a global search, but it is easy to produce the shock phenomenon and affect the overall performance of the algorithm. The try_ number is bigger, the following behavior and cluster behavior are more prominent, convergence speed is faster; on the contrary, the try_ number is smaller, the random behavior of fish more prominent, so the algorithm can search the global optimal solution in 
a wider range, but the prominent random behavior can cause value of fitness shock, it is not conducive to the local search. In view of the above question, this article through the introduction of adaptive parameter adjustment and membrane computing theory, proposed an improved artificial fish swarm algorithm based on membrane computing.

This paper presents a diversity factor $\alpha$, it expresses in the proportion of same individual in fish swarm, with the rate of change to control the try_ number, step and perspective.

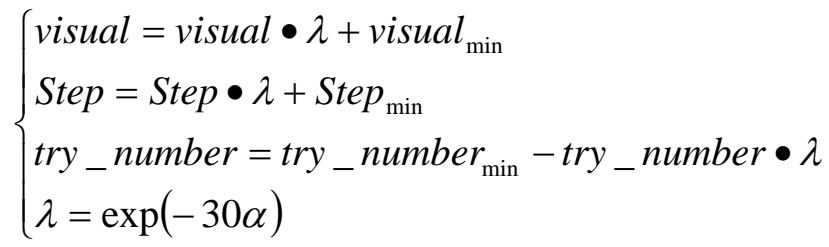

\subsection{The algorithm flow}

Step1: population initialization, generating a hierarchy of two layers from inside to outside: membrane 1and membrane 2, every membrane contains a fish;

Step2: Each individual artificial fish of membrane 1 followed by the implementation of foraging, cluster, rear end behavior, according to (1) to update their position;

Step3: According to the rules of communication judge whether carry the fish into the membrane 2, update the populations of membrane 2;

Step4: Each individual artificial fish of membrane 2 followed by the implementation of foraging, cluster, rear end behavior, according to (1) to update their position;

Step5: According to the rules of the exchange to determine whether carry the fish into the film 1 , update the populations of membrane 1 ;

Step6: Judgment termination condition. Achieve a prescribed number of iterations, then stop the operation, otherwise go back to step Step2.

\section{The analysis of simulation}

Compared with the algorithm of this paper and artificial fish swarm algorithm, it is used to verify the effectiveness of the algorithm. In this paper, the algorithm parameter settings are as follows: the horizon of visual is 4, the minimum viewing distance of min_visual is 1, step is 3, the min_step is 1 , repeated attempts try_number is 3, the minimum repeated attempts min_try_number is 2 , the fish scale is 50.In the experiment, flow shop need process 12 works, each job must pass 3 processes, parallel processing machines of each procedure are $3,2,2$, the processing capacity of each machine is different, the specific processing time as shown in table 1 . Table 2 is the running results of this algorithm and artificial fish swarm algorithm each running 10 times.

Table 1 The processing time of all works on the machines

\begin{tabular}{|c|c|c|c|c|c|c|c|}
\hline Works & Machine1 & Machine2 & Machine3 & Machine4 & Machine5 & Machine6 & Machine7 \\
\hline 1 & 3 & 4 & 2 & 3 & 2 & 4 & 3 \\
\hline 2 & 3 & 2 & 4 & 2 & 2 & 3 & 3 \\
\hline 3 & 2 & 1 & 2 & 3 & 3 & 2 & 2 \\
\hline 4 & 2 & 2 & 2 & 3 & 4 & 3 & 3 \\
\hline 5 & 3 & 2 & 2 & 4 & 2 & 3 & 2 \\
\hline 6 & 2 & 4 & 3 & 3 & 4 & 4 & 2 \\
\hline 7 & 2 & 2 & 2 & 3 & 2 & 4 & 2 \\
\hline 8 & 2 & 2 & 3 & 4 & 2 & 4 & 3 \\
\hline
\end{tabular}


Table2 10 times results of the algorithm and artificial fish swarm algorithm

\begin{tabular}{|c|c|c|c|c|c|c|c|c|c|c|c|}
\hline & Numbers & 1 & 2 & 3 & 4 & 5 & 6 & 7 & 8 & 9 & 10 \\
\hline $\begin{array}{c}\text { the } \\
\text { algorithm } \\
\text { of the } \\
\text { paper }\end{array}$ & $\begin{array}{c}\text { The } \\
\text { value of } \\
\text { the } \\
\text { objective } \\
\text { function }\end{array}$ & 22 & 22 & 22 & 22 & 23 & 22 & 22 & 23 & 23 & 22 \\
\hline $\begin{array}{c}\text { artificial } \\
\text { fish } \\
\text { swarm } \\
\text { algorithm } \\
\text { value of } \\
\text { the } \\
\text { objective } \\
\text { function }\end{array}$ & 22 & 23 & 23 & 23 & 22 & 23 & 22 & 22 & 22 & 22 \\
\hline
\end{tabular}

The change trend of fitness with generations is as shown in Figure 1. We can see from Figure 1 obviously, this algorithm of this paper can quickly converge to the optimal value.

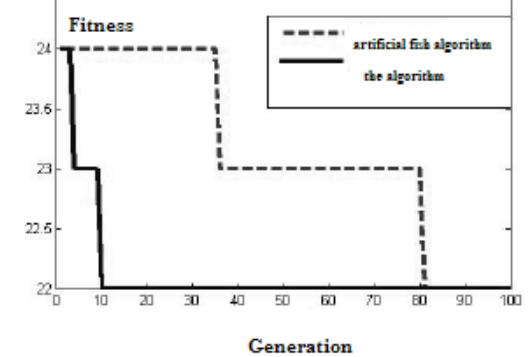

Fig.1 The change trend of fitness with generations

\section{Conclusions}

This paper proposes an artificial fish swarm algorithm based on membrane computing. The algorithm is applied to solve permutation flow shop scheduling problem, the performance have been improved obviously. Experiments show that: the algorithm is feasible and effective for solving permutation flow shop scheduling problem.

\section{References}

[1] Li Xiaolei, Shao Zhijiang, Jixin Qian. An animal autonomous body optimization model: fish swarm algorithm [J]. Systems engineering theory \& practice, 2002, 22 (11): 32-38.

[2]MAES P. From animals to animals 4: Proceedings of the fourth international conference on simu lation of adaptive behavior [M]. The MIT Press, 1996.

[3] Wang Lianguo, Hong Yi, Zhao Fuqing, Yu Dongmei. A simplified artificial fish swarm algorithm [J]. Mini micro system, 2009, 8 (8): 1663-1667.

[4] Liu Yanjun, Jiang Mingyan. An improved artificial fish swarmalgorithm based on adaptive vision and step [J]. Computer engineering and application, 2009, 45 (25): 35-37.

[5] Fan Yujun, Wang Dongdong, Sun Mingming. Improved artificial fish swarm algorithm [J]. Journal of Chongqing Normal University (NATURAL SCIENCE EDITION), 2007, 24 (3): 23-26.

[6] Huang Liang. Membrane computing optimization method research [D]. Doctoral dissertation of Zhejiang University, 2007. 